\section{Erfolgreiche ambulante Adipositas-Therapie}

W er bei Kindern und Jugendlichen eine Gewichtsreduktion erreichen will, muss langfristig planen. Für nachhaltige Erfolge soll die gesamte Familie einbezogen werden. „Wir sind uns bei der Adipositas heute relativ sicher, dass multimodale Lifestyle-Programme mit Elternbetreuung einen Effekt haben, wenn sie in eine langfristige Behandlungskette eingebettet sind“, konstatierte PD Dr. Susanna Wiegand, Berlin. Die Sprecherin der Arbeitsgemeinschaft Adipositas im Kindes- und Jugendalter stellte Bedingungen vor, unter denen eine ambulante Adipositas-Therapie gelingen kann.

Schon in früheren Untersuchungen war deutlich geworden, wie wichtig eine Familienbeteiligung sowie der Einsatz multiprofessioneller Teams für den Therapieerfolg sind. Wiegand und Kollegen hatten die Voraussetzungen für einen nachhaltigen Gewichtsverlust an über 3.000 Patienten untersucht. Im ZweiJahres-Verlauf zeigte sich, dass es Mädchen schwerer haben als Jungen, langfristig abzunehmen, und dass sich Jüngere leichter tun als Ältere. Besondere Schwierigkeiten hatten Kinder mit Migrationshintergrund sowie extrem Adipöse. Verlaufsdokumentationen zahlreicher Behandlungszentren bestätigen zudem: In einem multiprofessionellen ambulanten Adipositas-Programm über bis zu vier Jahre haben Kinder ohne Migrationshintergrund und mit voll berufstä- tigen Eltern die besten Chancen auf dauerhafte Gewichtsreduktion. Bekannt ist auch, dass sich diejenigen besonders schwer tun, die viel metabolisch relevantes Fettgewebe, also einen großen Bauchumfang haben. Bereits bei der Erstvorstellung bestand bei $60 \%$ der extrem adipösen Kinder mindestens eine Komponente des metabolischen Syndroms, so Wiegand. Es hätte sich aber gezeigt, dass sich im Rahmen einer ambulanten Adipositas-Therapie durch Bewegung sämtliche Komponenten signifikant verbesserten, selbst wenn der BMI stabil bleibe.

Aus neueren Studiendaten wisse man zudem, so Weigand, dass sich ein gestör- ter Tag-Nacht-Rhythmus, eine späte Zubettgehzeit sowie erhöhter Medienkonsum negativ auf den BMI auswirkten.

Zumindest die extreme Adipositas mit Komorbiditäten müsse als chronische Erkrankung gesehen werden, forderte Wiegand. Die Wunschvorstellung sei, dass der Pädiater die Patienten einem ambulanten oder stationären Programm zuweise und danach wieder niedergelassene Kollegen übernähmen. Parallel dazu sei eine Elternbetreuung erforderlich. Nur durch eine solche Behandlungskette könne der langfristige Erfolg einer Adipositastherapie gesichert werden.

Dr. Christine Starostzik

Wiegand S. Adipositas - Wann hilft ambulante Therapie?

\title{
Wann hilft eine stationäre Adipositas-Reha?
}

Eine stationäre Reha-Maßnahme ist nur dann wirksam, wenn sie Baustein einer Langzeitbetreuung ist. Dr. Rainer Stachow von der Fachklinik Sylt stellte die Ergebnisse eines Nachuntersuchungsprojekts der Deutschen Rentenversicherung mit 1.241 Kindern und Jugendlichen nach Adipositas-Reha vor. Sechs Wochen nach Ende der Maßnahme zeigten sich für fast alle Patienten erhebliche Verbesserungen bei BMI, Krankheitsmanagement, Blutdruck und Lebensqualität. Während am Reha-Ende mehr als 70\% ihren BMI erfolgreich reduziert hatten, konnten dies ein Jahr später nur noch $16 \%$ nachweisen. Zudem waren mehr als 70\% der Reha-Teilnehmer für die Nachuntersuchung nicht mehr erreichbar. Mit einer ambulanten multiprofessionellen Weiterbetreuung, so Stachow, bleibe dagegen auch ein Jahr nach der Reha der BMI noch signifikant reduziert. Darüber hinaus sei für den Erfolg der stationären Maßnahme die ambulante Vorbereitung wichtig. Nach Reha-Ende sollten sich die Patienten mindestens einmal pro Quartal in der Praxis vorstellen, um unter anderem Schwierigkeiten aufzuspüren und aus dem Weg zu räumen. Auch ein Hausbesuch sei informativ, um das Lebensumfeld beurteilen zu können.

Stachow R. Wann hilft eine Rehaklinik?

\section{Pauschale Intelligenztests bringen nichts}

U m die Fähigkeiten von Kindern mit Intelligenzminderung richtig abzubilden, ist die Auswahl des geeigneten Testverfahrens besonders wichtig. „Intelligenz ist keine Eigenschaft, sondern ein Funktionszustand. Eine Störung der Intelligenzentwicklung steht im Kontext mit bestimmten Lebenssituationen“, erklärte Dr. Friedrich Voigt aus München. Intelligenzquotienten (IQ) unter 70 oder 68 Punkten werden bislang als Grenzlinie zu einer Störung beschrieben. Werte unter 40 oder 50 sind mit Intelligenztests meist nicht mehr messbar, und eine zuverlässige Diagnostik ist ohnehin erst ab einem Alter von vier bis fünf Jahren möglich.

Doch die Standarddefinitionen wanken, denn im letzten Jahrzehnt habe sich das Verständnis der Intelligenz von Kindern gewandelt, sagte Voigt. Im neuen ICD-11 werde man sich deshalb auch von den heute gültigen IQ-Grenzen verabschieden. Die Schwierigkeit bei der Intelligenzmessung sei, dass sich die Normalverteilung immer auf gleichaltrige Kinder beziehe. So werde beispielsweise ein 12-jähriges Kind mit Tri- somie 21 mit Kindern verglichen, die in die fünfte oder sechste Klasse gehen. Ist das Kind aber auf der Entwicklungsstufe eines 4- oder 5-Jährigen, mache es vielleicht gar keinen Sinn, diesen Test anzuwenden, fügte Voigt hinzu. Um Stärken und Schwächen in relevanten Teilbereichen zu erfassen, müsse vor der Wahl des geeigneten Tests bei normal entwickelten wie bei Kindern mit Intelligenzstörungen ein vorläufiges Entwicklungsprofil erstellt werden.

Dr. Christine Starostzik

Voigt F. Störungen der Intelligenzentwicklung: psychologische Diagnostik 